Revista de Psicología de la PLCP. Vol. XVI, 1,1998.

\title{
LA IMAGEN MENTAL EN EL SISTEMA DE ENLACE
}

\author{
Alfredo Campos y Clara Isabel Fernández \\ Universidad de Santiago de Compostela
}

Jit articulo estudia a través de cuatro experimentos la influencia de la capacidad de los sujetos para format imágenes y del tipo de imagen que utilizan, en el recucrdo serial inmediato y retardo de palabras. En los experimentos 1 y 2 se analiza la influencia de estas variables en el recuerdo serial inmediato de listas cortas (16 items) y largas (28 items), y en los experimentos 3 y 4 sc investiga h influencia de las mismas variables en el recuerdo scrial retardado (una semann). La capacidad de los sujetos de formar imágenes, medida a través de la Escala Lspacial del Test de Aptitudes Mentales Primarias, influyó en el recuerdo scrial inmediato de listas largas. El tipo de imagen (normal y rara) influyó en el recuerdo inmediato serial de listas cortas. Lin todos los experimentos se encontro diferencia entre los sujetos que utilizaron imágenes mentales y los que no las utilizaron.

Palabras claves: Imasen mental, recuerdo, memoria, sistema de enlace.

\section{Mental imagery in link system}

Four cxperiments were carried out to explore the influence of imaging capacity and image type (nomal or bizarre) on immediate and delayed recall of word lists. Experiments 1 and 2 studied immediate serial recall of 16 - and 28 -item word lists, and experiments 3 and 4 studicd serial recall of 16 - and 28 - item word lists one week after their presentation. Imaging capacity, as measured by the Space Scale of the Primary Mental Abilities Test, affected immediate recall of long lists. Image type affected immediate recall of short lists. In four experiments there was a significant difference between the performance of subjects who used mental imagery for recall and those who did not.

Key words: Mental imagery, recall, memory, link system.

1. Departamento de Psicología Básica 15706 Santiago de Compostela, España. Correo electrónico: pscampos@uscmail.usc.es 
La utilización de las imágenes mentales en el aprendizaje produce, según todas las investigaciones, un efecto positivo, superior al que se obtiene mediante otros métodos de aprendizaje (Denis, 1984). Bower y Reitman (1972) presentaron pares de palabras a diferentes grupos para que las memorizasen en formas diferentes: repitiendo las palabras, formando imágenes de cada palabra, y formando imágenes en interacción. Los que se imaginaron cada ítem formando las imágenes juntas e interactuando entre ellas, mostraron un recuerdo mayor para los pares de palabras que los otros grupos. El grupo que utilizó imágenes separadas tuvo un mayor recuerdo que el grupo que utilizó el método de la repetición.

Las imágenes en interacción son más eficaces para el recuerdo que las imágenes independientes (Bergfeld, Choatc y Kroll, 1982; Campos y Fernández, 1995a; Kroll, Schepeler y Angin, 1986). Lutz y Lutz (1977) encontraron que se recordaba mejor una marca publicitaria cuando ésta aparecía en una misma imagen con el producto, que cuando se presentaban como dos imágenes separadas. Probablemente, una de las razones de la efectividad de las imágenes en interacción es que las imágenes de elementos separados pueden combinarse en una sola imagen que se recuerda como una unidad. En consecuencia, cada uno de los fragmentos de la imaginación sirve de clave para recordar el resto de la unidad (Begg, 1982; Kosslyn, 1986).

Si bien todos los autores están de acuerdo en la eficacia de la interacción, no sucede lo mismo con las imágenes raras. A pesar de que los autores recomiendan la utilización de imágenes raras en el aprendizaje (Higbee, 1991), los estudios experimentales no son contundentes. Algunos encuentran una superioridad con la utilización de imágenes normales, otros 
no encuentran diferencias entre ambos tipos de imágenes, y finalmente, otros encuentran las imágenes raras superiores a las normales (Bergfeld, Choate y Kroll, 1982; Campos y Fernández, 1995b; Emmerich y Ackerman, 1979; Pra Baldi, De Beni, Cornoldy y Cavedon, 1985).

La aparente contradicción de resultados se debe, fundamentalmente, a la utilización de metodologías diferentes. Estas diferencias metodológicas se deben, en gran medida, al tipo de recuerdo utilizado (recuerdo inmediato, recuerdo retardado), al tipo de tarea (recuerdo de pares asociados y recuerdo libre), y al tipo de diseño utilizado (listas puras y listas mixtas) (Campos y Fernández, 1995b; Kroll, Jaeger y Dornfest, 1992; Pérez y Campos, 1997; Riefer y Rouder, 1992).

Se puede afirmar que la capacidad que tienen los sujetos para formar imágenes influye en el recuerdo (Campos y Fernández, 1995b; Denis, 1982; O'Brien y Wolford, 1982; Zoller, Workman y Kroll, 1989), aunque esta influencia disminuye si el recuerdo se da al cabo de una semana (Campos y Fernández, 1995b; Zoller, Workman y Kroll, 1989), o si se trabaja con material abstracto (Denis, 1982).

En la presente investigación se intentó, a través de una serie de cuatro experimentos, aportar luz a la controversia sobre el recuerdo serial mediante el sistema de enlace con listas cortas/largas, recuerdo inmediato/retardado, imagen normal/rara. Se utilizó estricto control sobre los sujetos, asegurándose que se cumpliesen las instrucciones respecto al uso de algún tipo de imagen.

\section{Experimento 1}

Aunque la mayoría de las investigaciones sobre aprendizaje serial mediante imágenes se han realizado con listas cortas (alrededor de 16 items) y con recuerdo inmediato, como se ha señalado anteriormente, los resultados mo son definitivos. Por esa razón, en el primer experimento se analizó la tinfluencia de la capacidad de imagen de los sujetos (medida a través de una prueba de rendimiento y de un cuestionario) y el tipo 
La imagen mental en el sistema de enlace

de imagen utilizada (normal, rara y sin imagen) en el recuerdo inmediato de listas cortas.

\section{Metodología}

\section{Participantes}

La muestra estaba formada por 310 estudiantes (132 mujeres y 178 varones) de Enseñanza General Básica, con una media de edad de 12 años, y un rango de 10 a 14 años. Este grupo de estudiantes fue el que permaneció después de que dos jueces rechazaran a 62 alumnos por no haber seguido las instrucciones. Se seleccionó al azar distintos centros de la ciudad de Orense (España), y también al azar, se asignó los participantes para cada grupo.

\section{Instrumentos}

Para medir la capacidad de los participantes para formar imágenes, se utilizó dos pruebas: el Vividness of Visual Imagery Questionnaire (VVIQ) (Marks, 1973), y la Escala Espacial del Test de Aptitudes Mentales Primarias (PMA) (Thurstone y Thurstone, 1989). Altas puntuaciones en el VVIQ indicaban poca claridad de imagen, y viceversa. Hemos utilizado dos pruebas diferentes (prueba de rendimiento y cuestionario) para medir la capacidad de los sujetos para formar imágenes porque las dos se consideraron medidas de imagen, y entre ellas existe una baja correlación (Campos y González, 1992a).

Asimismo, se confeccionó tres listas de 16 palabras cada una. La mitad de las palabras de cada lista tenía una imagen poco clara, como desinterés, realismo, lealtad, decisión, y la otra mitad eran palabras con claridad superior, como águila, manzana, pastel, elefante. Las palabras fueron tomadas de estudios previos realizados por Campos (1989, 1993), Campos y Astorga (1986), Campos y Gonzáles (1992b). De igual manera se elaboró un cuestionario para registrar el tipo de estrategia utilizado para 
memorizar la lista de palabras. En el cuestionario aparecían todas las palabras y se preguntaba el tipo de imagen utilizada, por cada par de palabras. El cuestionario también tenía una línea en blanco para que el participante describiese la imagen.

\section{Procedimiento}

Se formó al azar tres grupos de estudiantes. A todos ellos se les dijo que se les iba a presentar una lista de palabras y que debían recordarlas en el mismo orden. Las palabras se presentaron mediante un proyector de diapositivas con temporizador a grupos de aproximadamente 30 estudiantes, a un ritmo de una palabra cada 15 segundos. A todos los alumnos se les presentó una lista de 16 palabras (lista corta).

A dos de estos grupos se les entrenó en el método de enlace para recordar las palabras, uniendo en una misma escena la imagen de una palabra con la imagen de la siguiente (A-B, B-C, C-D, etc.). Un grupo utilizó imágenes normales (por ejemplo, para recordar las palabras águila y manzona, se pucde imaginar un águila volando que lleva una manzana en el pico), otro (yrupo usó imágenes raras (por ejemplo, un águila que tiene cabeza de águila y cuerpo de caballo que lleva metida en su pata derecha delantera una manzana hueca), y el tercer grupo no utilizó imágenes (sólo pensó en las palabras águila y manzana). Los tres grupos debían recordar, una vez finalizada la presentación de la lista, el mayor número posible de palabras en el orden en que habian sido presentadas.

A continuación los alumnos respondieron el cuestionario en el que se les pedía señalar el tipo de estrategia utilizada (imagen normal, rara, sin imagen) para cada par de palabras, y que la describiesen en una frase. Estas frases fueron analizadas por dos jueces. Los estudiantes que no cumplieron con las instrucciones fueron rechazados. Se consideró que se había cumplido con las instrucciones cuando, según la opinión de dos jueces (acuerdo entre ellos de .9), se había seguido las indicaciones, por lo menos en la mitad de las respuestas. Posteriormente a los participantes se presentó el VVIQ y el PMA. 
La imagen mental en el sistema de enlace

\section{Resultados}

La media de recuerdo obtenida por cada grupo se muestra en el Cuadro 1. Para averiguar si la diferencia entre los grupos era significativa, se efectuó un Análisis de Varianza (ANOVA) de 2 (PMA) x 2 (VVIQ) x 3 (Tipo de imagen). Sólo se encontró significativa la influencia del tipo de imagen $(F(2,298)=134.34, p<.001)$. El análisis Scheffé registró diferencias significativas entre los tres grupos en el recuerdo inmediato de las listas cortas.

\section{Cuadro 1}

Media (M) del Recuerdo Inmediato de Listas Cortas para cada Grupo

\begin{tabular}{|c|c|c|}
\hline Gmpo & $\begin{array}{c}M \\
\text { de recuerda }\end{array}$ & $n$ \\
\hline \multicolumn{3}{|l|}{ PMA } \\
\hline Puntuación Alta & 8.19 & 129 \\
\hline Puntuación Baja & 7.39 & 181 \\
\hline \multicolumn{3}{|l|}{ VVIQ } \\
\hline Puntuación Alta & 7.10 & 142 \\
\hline Puntuación Baja & 8.24 & 168 \\
\hline \multicolumn{3}{|c|}{ Tipo Imagen } \\
\hline Ausente & 5.15 & 127 \\
\hline Normal & 10.2 & 125 \\
\hline Rara & 8.40 & 58 \\
\hline
\end{tabular}

\section{Experimento 2}

Cuando las listas tienen pocos ítems (alrededor de diez o menos) puede suceder que el uso de imágenes no sea superior a cualquier otro método de recuerdo (Higbee, 1991). Según Bellezza (1981), cuando las listas tienen pocos ítems, las personas confian en su memoria y no utilizan estrategias de imagen; en cambio, cuando la lista es larga se ven obligados a utilizar las imágenes, por eso, en el siguiente experimento se estudió la influencia de la capacidad de los sujetos para formar imágenes (medidas 
a través del PMA y del VVIQ) y el tipo de imagen utilizada (normal, rara, sin imagen) en el recuerdo inmediato de listas largas.

\section{Metodología}

\section{Participantes}

La muestra estaba conformada, en un primer momento, por 402 estudiantes. Después de que los jueces rechazaron a 67 , quedó reducida a 335 (159 mujeres y 176 varones). Ellos tenían las mismas características que los participantes del primer experimento. Los materiales y procedimientos también se mantuvieron, pero se usó listas largas (28 palabras) para que los participantes memoricen.

\section{Resultados}

Las medias de recuerdo de cada grupo se encuentran en el Cuadro 2. Para averiguar la influencia de cada variable sobre el recuerdo, se efectuó un Análisis de Varianza (ANOVA) de 2 (PMA) x 2 (VVIQ) x 3(Tipo de imagen). Todas las variables influyeron significativamente, PMA ( $F$ $(1,323)=8.339, p<.01), \operatorname{VVIQ}(F(1.323)=5.234, p<.05)$, tipo de imagen $(F(2.323)=81.228, p<.001)$. Los análisis Scheffé indicaron que existian diferencias significativas entre el grupo sin imágenes y los que utilizaron imágenes normales o raras; sin embargo, no se encontró diferencias significativas entre los grupos que usaron imágenes normales y raras.

\section{Experimento 3}

La mayoría de las investigaciones que estudian la eficacia de la mnemotecnia analizan el recuerdo inmediato. Aunque algunos estudios han mostrado que la mnemotecnia no es útil para la retención a largo plazo, otros estudios han demostrado su eficacia (Bellezza, 1981; Roediger III, 1980; Straub y Granaas, 1992). En este experimento se intentó analizar 
La imagen mental en el sistema de enlace

\section{Cuadro 2}

Media M) del Recuerdo Inmediato de Listas Largas para cada Grupo

\begin{tabular}{|c|c|c|}
\hline Grupo & $\begin{array}{c}M \\
\text { de recuerdo }\end{array}$ & $n$ \\
\hline \multicolumn{3}{|l|}{ PMA } \\
\hline Puntuación Alta & 10.25 & 162 \\
\hline Puntuación Baja & 8.89 & 173 \\
\hline \multicolumn{3}{|l|}{ VVIQ } \\
\hline Puntuación Alta & 8.54 & 142 \\
\hline Puntuación Baja & 10.30 & 193 \\
\hline \multicolumn{3}{|c|}{ Tipo Imagen } \\
\hline Ausente & 6.26 & 140 \\
\hline Normal & 12.08 & 145 \\
\hline Rara & 11.42 & 50 \\
\hline
\end{tabular}

la influencia de la capacidad de los participantes para formar imágenes (PMA y VVIQ) y el tipo de imagen (normal, rara, sin imagen) utilizada en el recuerdo retardado de listas cortas, siempre controlando que los sujetos sigan las instrucciones.

\section{Metodología}

La muestra quedó reducida a 300 estudiantes (123 mujeres y 177 varones), después de que los jueces rechazaran a 58. La forma de selección y las características de los participantes fueron las mismas que en el experimento 1. También se siguió el mismo procedimiento y se utilizó el mismo material que en el primer experimento, pero los participantes debían recordar al cabo de una semana. A los alumnos se les indicó que aprendiesen la lista de palabras, pero no se les dijo que se les preguntaría dentro de una semana. Al finalizar la presentación de los ítems, los estudiantes respondieron a los cuestionarios, excepto al test de recuerdo, que se les presentó después de una semana. 


\section{Resultados}

En el Cuadro 3 se encuentran las medias de recuerdo obtenidas por los distintos grupos. Para analizar si la diferencia existente entre los grupos era significativa, se efectuó un Análisis de Varianza (ANOVA) de 2 (PMA) x 2 (VVIQ) $\times 3$ (tipo de imagen). Encontramos un efecto significativo de la claridad de imagen, medida por el VVIQ $(F(1,288)=12.406, p<$ $.001)$, y del tipo de imagen $(F(2,288)=19.802, p<.001)$. Los análisis de Scheffé mostraron diferencias significativas entre el grupo sin imagen y cada uno de los otros dos; sin embargo, entre el grupo de imagen normal y el grupo de imagen rara no se encontraron diferencias significativas.

\section{Cuadro 3}

Media (M) del Recuerdo Retardado de Listas Cortas para cada Grupo

\begin{tabular}{|lcc|}
\hline Grupo & $M$ & $n$ \\
\hline PMA & de recuerdo & \\
Puntuación Alta & & 171 \\
Puntuación Baja & 5.04 & 129 \\
$\quad$ VVIQ & 4.51 & \\
Puntuación Alta & & 140 \\
Puntuación Baja & 4.25 & 160 \\
$\quad$ Tipo Imagen & 5.30 & \\
Ausente & & 119 \\
Normal & 3.70 & 124 \\
Rara & 5.64 & 57 \\
\hline
\end{tabular}

\section{Experimento 4}

La efectividad de la mnemotécnica de imágenes en el recuerdo retardado es discutida por algunos autores, a pesar de que en muchos experimentos se ha observado resultados positivos. Todavía es más 
discutida esta efectividad cuando se utilizan listas largas de palabras en el recuerdo retardado. Por eso, en el último experimento se analizó la influencia de la capacidad de los sujetos para formar imágenes (PMA y VIQ) y el tipo de imagen (normal, rara y sin imagen) empleada en el recuerdo serial retardado de listas largas.

\section{Metodología}

La muestra, que tenía las mismas características que en el experimento 1, estaba compuesta por 378 estudiantes, pero se redujo a 314 (136 mujeres y 178 varones), después de que los jueces rechazaron a 64. Los materiales y procedimientos fueron los mismos, excepto que los alumnos debían recordar listas largas (28 palabras) después de una semana.

\section{Resultados}

Las medias de recuerdo retardado se aprecian en el Cuadro 4. Se efectuó un Análisis de Varianza (ANOVA) de 2 (PMA) x 2 (VVIQ) x 3 (tipo de imagen), y se encontró que sólo el tipo de imagen influyó significativamente en el recuerdo retardado de listas largas $(F(2,302)=$ $16.958, p<.001)$. El análisis Scheffé encontró diferencia en el recuerdo entre el grupo sin imagen y el que utilizó imagen normal o imagen rara; sin embargo, no se encontró diferencia entre el recuerdo del grupo que empleó imagen normal y el que empleó imagen rara.

\section{Discusión}

La primera conclusión clara que se obtiene de los resultados es que existe diferencia en el recuerdo serial de palabras cuando se usan imágenes mentales y cuando no se usan (ya sea en listas cortas como largas, tanto en el recuerdo serial inmediato como en el retardado), lo que coincide con el grueso de las investigaciones sobre el tema (Higbee, 1991). 


\section{Cuadro 4}

Media (M) del Recwerdo Retardado de Listas Largas para cada Grupo

\begin{tabular}{|c|c|c|}
\hline Grppo & $\begin{array}{c}M \\
\text { de recuerdo }\end{array}$ & $n$ \\
\hline \multicolumn{3}{|l|}{ PMIA } \\
\hline Puntuación Alta & 5.86 & 178 \\
\hline Puntuación Baja & 5.20 & 136 \\
\hline \multicolumn{3}{|l|}{ VVIQ } \\
\hline Puntuación Alta & 4.85 & 130 \\
\hline Puntuación Baja & 6.08 & 184 \\
\hline \multicolumn{3}{|c|}{ Tipo Imagen } \\
\hline Ausente & 3.92 & 121 \\
\hline Normal & 6.32 & 130 \\
\hline Rara & 7.21 & 63 \\
\hline
\end{tabular}

El tipo de imagen (normal/rara) sólo influyó en el recuerdo inmediato de listas cortas, siendo superior el recuerdo con imágenes normales. En los demás experimentos no se ha encontrado influencia del tipo de imagen. En todos los experimentos, excepto en el último (recuerdo retardado de listas largas), se observa una diferencia entre las medias a favor de la imagen normal, si bien ninguna diferencia es significativa, excepto en el recuerdo inmediato de listas cortas. Probablemente sea en esta situación en la que es mayor el efecto de la imagen normal. Estos resultados están en la línea de aquellos encontrados por otros investigadores (Bellezza, 1983a,b; Campos y Fernández, 1995b; Roediger III, 1980).

La capacidad de los sujetos para formar imágenes, medida por el VVIQ, influyó en el recuerdo inmediato de listas largas, y en el recuerdo retardado de listas cortas. No influyó cuando las listas eran cortas y el recuerdo era inmediato, probablemente porque la tarea era fácil y no se necesitaba esfuerzo en exceso (poner en práctica su capacidad) (Belleza, 1981). Asimismo, no influyó en el recuerdo retardado con listas largas 
porque, probablemente los participantes desistieron ante el esfuerzo de recordar, al cabo de una semana, una cantidad tan grande de palabras. Las medias de recuerdo retardado de listas largas mediante imágenes son bajas, si bien son superiores a las obtenidas con ausencia de imágenes. Estos resultados están en la línea de los obtenidos por Denis (1982), O'Brien y Wolford (1982) y Zoller, Workman y Kroll (1989).

De estos cuatro experimentos se puede deducir que es necesario efectuar un control sobre los participantes cuando se dan las instrucciones de formar imágenes, dado el gran número de personas que se tuvo que rechazar. Si en la presente investigación no se hubiese efectuado un control sobre el cumplimiento de las instrucciones, los resultados se hubieran contaminado.

En el recuerdo serial de palabras se debe utilizar imágenes mentales, ya que el recuerdo es superior a cuando no se utilizan, tanto cuando el recuerdo es inmediato como a largo plazo. Cuando las listas son largas y el recuerdo es inmediato también influyc con claridad la capacidad para formar imágenes.

Se recomienda nuevas investigaciones que, siguiendo el control metodológico empleado en la presente investigación y rechazando a los participantes que no sigan las instrucciones, se orienten a analizar la eficacia de la imagen en distintas situaciones (pares asociados, palabras independientes, palabras con poca imagen o mucha imagen, recuerdo, reconocimiento). De este modo, se podrá aplicar la estrategia más adecuada a cada situación de aprendizaje.

\section{Referencias}

Begg, I. (1982). Imagery, organization and discriminative processes. Canadian Journal of Psychology, 36, 273-290.

Bellezza, F.S. (1981). Mnemonic devices: Classification, characteristics and criteria. Review of Educational Research, 51, 247-275. 
Bellezza, F. S. (1983a), Mnemonic-device instruction with adults. En M. Pressley y R. J. Levin (Eds.), Cognitive strategy research: Psychological foundations (pp. 51-73). Nueva York: Springer-Verlag.

Bellezza, F. S. (1983b). The spatial-arrangement mnemonic. Joumal of Educational Psycholog), 75, 830-837.

Bergfeld, V. A., Choate, L. S. y Kroll, N. E. A. (1982). The effect of bizarre imagery on memory as a function of delay: A reconfirmation of interaction effect. Joumal of Mental Imagery, 6, 141-158.

Bower, G. H. y Reitman, J. S. (1972). Mnemonic elaboration in multilist learning. Joumal of Verbal Learning and Verbal Behavior, 11, 478-485.

Campos, A. (1989). Emotional values of words: Relation with concreteness and vividness of imagery. Perceptual and Motor Skills, 69, 495-498.

Campos, A. (1993). Interrelación del valor de imagen de las palabras con otros valores de las mismas. Manuscrito no publicado. Universidad de Santiago de Compostela.

Campos, A. y Astorga, V. M. (1986). Spanish, North American and Canadian ratings of imagery values of words. Perceptual and Motor Skills, 63, 889-890.

Campos, A. y Fernández, C. I. (1995a). Imágenes interactivas y recuerdo serial de palabras. Revista Galega de Psicopedagoxia, 12, 167-172. Campos, A. y Fernández, C. I. (1995b). Imagen mnemónica y recuerdo de series de palabras. Adaxe, 11, 27-33.

Campos, A. y González, M. A. (1992a). Repercusión de la autoimagen en el diagnóstico de la capacidad de formar imágenes mentales. Fistudios de Psicologia, 47, 27-33.

Campos, A. y González, M. A. (1992b). Imagery, concreteness, emotionality and meaningfulness values of words: Replication and extension. Perceptual and Motor Skills, 74, 691-696.

Denis, M. (1984). Las imágenes mentales. Madrid: Siglo XX1.

Emmerich, H. J. y Ackerman, B. P. (1979). A test of bizarre interaction as a factor in children's memory. Joumal of Genetic Psycbology, 134, 225 232.

Higbee, K. L. (1991). Su memoria. Cómo dominarla para recordar todo. Barcelona: Paidós Ibérica. 
La imagen mental en el sistema de enlace

Kosslyn, S. W. (1986). Capacidad para formar imágenes mentales. En R. J. Sternberg (Ed.), Las capacidades bumanas (pp. 195-224). Barcelona: Labor.

Kroll, N. E. A., Jaeger, G. y Dornfest, R. (1992). Metamemory for the bizarre. Joumal of Mental Imagery, 16, 173-190.

Kroll, N. E. A., Schepeler, E. M., y Angin, K. T. (1986). Bizarre imagery: The misremembered mnemonic. Joumal of Experimental Psycbologl: Learning, Memory and Cognition, 12, 42-53.

Lutz, K. A. y Lutz, R. (1977). Effects of interactive imagery on learning: applications to advertising. Joumal of Applied Psychology, 62, 493-498. Marks, D.F. (1973). Visual imagery differences in the recall of pictures. British Journal of Psychology, 64, 17-24.

O'Brien, E. J. y Wolford, C. L. R. (1982). Effect of delay in testing on retention of plausible versus bizarre mental images. Joumal of Experimental Psychology: Learning, Memory and Cognition, 8, 148-152.

Pérez, M. J. y Campos, A. (1997). Dificultades metodológicas en el diagnóstico del tipo de imagen utilizada en el aprendizaje. Revista Galego-Portuguesa de Psicoloxia e Educación, 1, 173-176.

Pra Baldi, A., De Beni, R., Cornoldy, C. y Cavedon, A. (1985). Some conditions for the occurrence of the bizarreness effect in free recall. British Joumal of Psychology, 76, 427-436.

Riefer, D. M. y Rouder, J. N. (1992). A multinomial modeling analysis of the mnemonic benefits of bizarre imagery. Memory and Cognition, 20, 601-611.

Roediger III, H. L. (1980). The effectiveness of four mnemonics in ordering recall. Journal of Experimental Psychology: Human Leaming and Menon;, 6, 558-567.

Straub, H. R. y Granaas, M. M. (1992). Interaction of instructions with the recall strategy actually used in a paired-associates learning task. Psychological Reports, 71, 987-993.

Thurstone, L. L. y Thurstone, T. G. (1989). Aptitudes Mentales Primarias. Madrid: TEA.

Zoller, C. L., Workman, J. S. y Kroll, N. E. (1989). The bizarre mnemonic: The effect of retention interval and mode of presentation. Bulletin of the Psychonomic Society, 27, 215-218. 